\title{
Healthcare System through Wireless Body Area Networks (WBAN) using Telosb Motes
}

\author{
Mr. Ather Parvez Abdul Khalil \\ Research Scholar, MSS's College of Engineering and Technology, Jalna, India \\ ather.ziya@gmail.com
}

\begin{tabular}{|c|c|}
\hline Article History & Abstract \\
\hline $\begin{array}{c}\text { Article Submission } \\
18 \text { January } 2012 \\
\text { Revised Submission } \\
20 \text { April } 2012 \\
\text { Article Accepted } \\
1 \text { May } 2012 \\
\text { Article Published } \\
\text { 30 June } 2012\end{array}$ & $\begin{array}{l}\text { Due to the increased demand for an effective communication technology in the health } \\
\text { care sector, a wireless network is needed that effectively monitors the patient's vital } \\
\text { sign and send reports concurrently to the remote system. This increases the comfort } \\
\text { level of monitoring the patients continuously. The main idea of this article work is to } \\
\text { build up a WBAN that endlessly monitors human healthiness parameters and reports } \\
\text { information to the nearby PC/sinks. The small wearable devices which is memory } \\
\text { efficient, energy efficient, less computational complexity are deployed on the patient to } \\
\text { be monitored. They self-configure to endlessly monitor critical signs such as heart beat, } \\
\text { core temperature etc. The energy efficiency and transmission delay is analyzed by using } \\
\text { Cooja simulator. Finally the design is implemented in the Telosb wireless sensor motes. } \\
\text { Keywords: Contiki OS, Telosb mote, Cooja, Sensors, WBAN }\end{array}$ \\
\hline
\end{tabular}

\section{Introduction}

Monitoring sick peoples automated is the great deal of the day. The worldwide elder people population will reach up to 761 million by the year 2025. Improvement in wireless based networks will decrease hospital stays for longer time, reduce the visiting frequency of doctors, efficient utilization of medical professionals and reducing cost. The advanced health care system provides early diagnosis of diseases and help the doctors to work from remote locations. Further, patient attending and prescription consultations can be rendered through wireless communication networks and channels. The omnipresent healthcare system can give a cheaper and one-touch way to manage patients distress from age oriented persistent diseases like heart disease as it require nonstop, long-term assessment rather than intermittent assessments.

\section{Existing Methodology}

The Institute of Electrical and Electronics Engineers (IEEE) confirmed the 802.15.4 IEEE standard in the year 2003. IEEE 802.15.4 comprises of the physical layer, superior MAC protocols leading to low speed Wireless Personal Area Network (WPAN). There is confusion everywhere between IEEE 802.15.4 with ZigBee, powered by ZigBee association. On the other hand, ZigBee utilizes the services rendered by IEEE 802.15.4 including peer-to-peer networks, mesh and cluster-tree networks leading to higher security. The embattled relevance for IEEE 802.15.4 are not limited to wireless sensor networks, home system automation \& networking, establishing connection between devices to PC and home related security requiring limited bit rates [3]. The physical layer offers bitrates of $20 \mathrm{kbps}$, $40 \mathrm{kbps}$ and $250 \mathrm{kbps}$ using $2.54 \mathrm{GHz}$ ISM band [8].Full function devices has a ability to communicate with other nodes of same network or they can communicate with the nodes of another network, they just acts as a bridge, they 
are also called pan-coordinator. Reduced functional devices only have the ability to communicate with the PANcoordinator.

\section{Design of WBAN Network}

In the proposed design of WBAN network, all the sensors are connected to the RFD devices and they forward the information to the coordinator. Coordinators have the ability to combine the data $\&$ to transmit it to sink. The mote which is connected to PC will be treated as sink, which transfers the received data to PC through USB cable.

The overall view of the hardware network setup is given. Three sensors that are interfaced with the motes and the sink and repeater motes has the following RIME addresses.

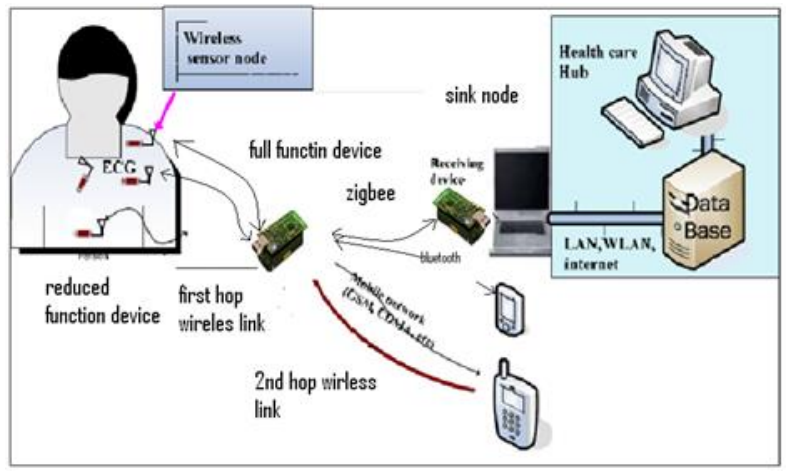

Fig 1. Proposed System Design

There are three kinds of motes used in the whole network. The first kind of mote is connected to the sensor which is in turn connected to the body of the patient. This mote collects the information from sensor and sends it to the nearby coordinator mote. The second kind of mote receives the sensed information from all motes and convert it into a single packet sends this information to the next mote which is connected with the computer. This mote can also serve as a repeater if the distance to be covered is large. This results in a multiple-hopping network where data is transferred from one mote to another mote. The number of intermediate motes depends upon the distance to be covered.

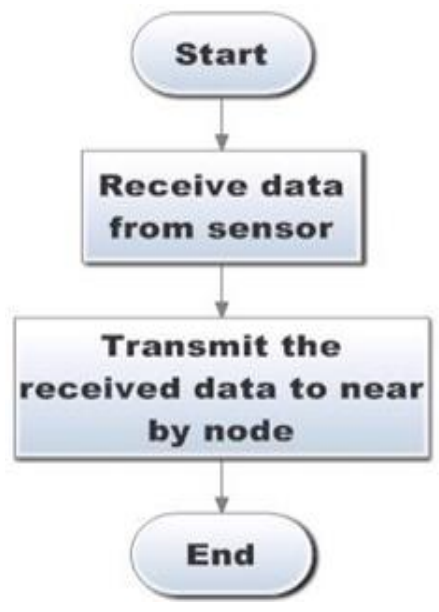

Fig 2. Flow chart of transmitter

ISSN: 2250-0839 


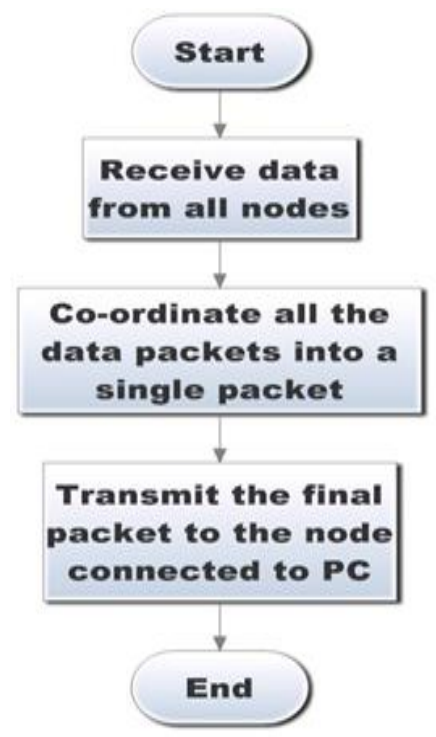

Fig.3: flow chart for coordinator

The last mote which is connected to the PC serves as a sink and displays the information about the patient collected from other motes on the computer screen. Based on the received information, the health status of the patient is monitored continuously

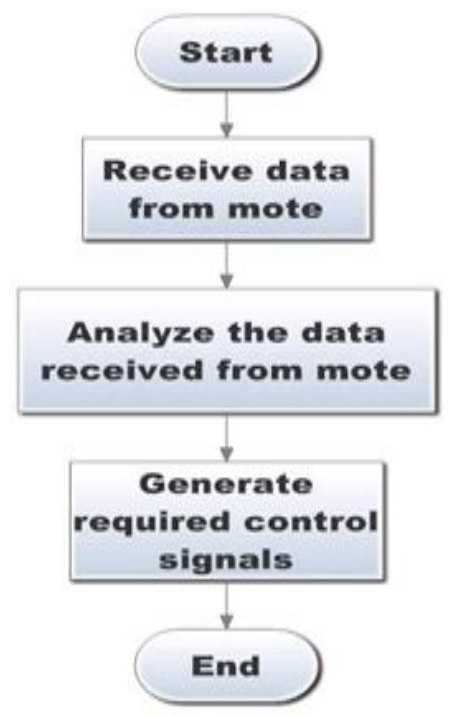

Fig 4. Flow chart of Receiver

\section{a. System Implementation Requirements}

The major hardware used in the work is the wireless sensor mote. There are several kinds of motes available. The mote used in the work is MEMSIC's TelosB Mote TPR2420. The detailed description of the mote and its features is given the next chapter. The other hardware used in this work is various sensors that monitor the patient's health condition. Several sensors like Heart beat monitoring, Motion detection sensor, Humidity sensors etc... is used in the work. 
The motes can be programmed using any RTOS which is supported by the selected hardware. Here the motes are programmed using a real time operating system called CONTIKI OS. This is an open source OS which is compatible with the given mote.

\section{b. Mote - Pc Communication}

Mote PC communication involves collecting some data from the mote through serial port of the computer. This happens in the last mote of the network which is connected to the Computer (sink). The Mote that acts as sink by collecting information from other the nearby repeater mote is connected to PC through USB extension cable.

\section{c. Mote - mote communication}

This communication involves the usage of Radio to transmit and receive packets among motes wirelessly. The mote which co-ordinates the packets from other motes to the sink mote use this type of communication. The Unicast type of message transfer takes place between motes. These motes are programmed to receive and transmit simultaneously and hence threads which run concurrently are used.

\section{d. Mote - Sensor Interfacing}

Different sensors are interfaced to motes using connecting wires. The data out pin of sensors are connected to any one of the Digital I/O pin of the processor. An event driven program is written for these kind of motes which continuously senses the pin to which sensor is connected. Once the pin goes HIGH, then it performs some calculations or directly transmits the information to the nearby repeater mote.

\section{Results and Discussion}

In the cooja simulator the network is simulated for RPL routing, to compare the results of practically achieved results and the results are tabulated. The simulated result is shown in figure 5.

Table 1: Power calculated at sink

\begin{tabular}{|c|c|c|c|c|c|}
\hline Parameter/mote & $\begin{array}{c}\text { Heart Beat } \\
\text { Mote }\end{array}$ & $\begin{array}{c}\text { Moisture Sensor } \\
\text { Mote }\end{array}$ & $\begin{array}{c}\text { PIR Sensor } \\
\text { Mote }\end{array}$ & $\begin{array}{c}\text { Repeater } \\
\text { Mote }\end{array}$ & $\begin{array}{c}\text { Sink } \\
\text { Mote }\end{array}$ \\
\hline $\begin{array}{c}\text { Overall power consumption } \\
\text { (maximum) }\end{array}$ & $50 \mathrm{~mW}$ & $59 \mathrm{~mW}$ & $59 \mathrm{~mW}$ & $111 \mathrm{~mW}$ & $59 \mathrm{~mW}$ \\
\hline Packet transmission delay & $26.24 \mu \mathrm{s}$ & $27.46 \mu \mathrm{s}$ & $26.24 \mu \mathrm{s}$ & $80 \mu \mathrm{s}$ & - \\
\hline Overall delay (seconds) & 10 & 1 & 1 & 10 & - \\
\hline $\begin{array}{c}\text { Range of operation } \\
\text { (outdoor range) }\end{array}$ & upto $75 \mathrm{~m}$ & Upto $75 \mathrm{~m}$ & Upto $75 \mathrm{~m}$ & Upto $75 \mathrm{~m}$ & $\begin{array}{c}\text { Upto } \\
75 \mathrm{~m}\end{array}$ \\
\hline $\begin{array}{c}\text { Range of operation } \\
\text { (indoor range) }\end{array}$ & upto $25 \mathrm{~m}$ & Upto $25 \mathrm{~m}$ & Upto $25 \mathrm{~m}$ & Upto $25 \mathrm{~m}$ & $\begin{array}{c}\text { Upto } \\
25 \mathrm{~m}\end{array}$ \\
\hline
\end{tabular}




\section{a. Overall delay calculation}

Overall delay associated in transmission $=$ packet transmission delay + data calculation time

Time consumption per sensing

PIR mote = sense and produce strings every second

Moisture mote $=$ sense and produce strings every second

Heart beat mot $=$ sense and produce data every 10 seconds.

\section{b. Total power calculation}

The repeater mote is at $2 / 3 \mathrm{rd}$ of a feet $(20 \mathrm{~cm})$ from the sensor motes (each).

The sink mote is 2 feets $(60 \mathrm{cms})$ from the repeater

Table 2. Packet Data Calculation

\begin{tabular}{|c|c|}
\hline \multicolumn{2}{|c|}{ Packet delay calculation } \\
\hline Theoretical data rate & $250 \mathrm{kbps}$ \\
\hline Practically achievable & $160 \mathrm{kbps}$ approx. \\
\hline
\end{tabular}

Table 3. Zigbee frame structure and size

\begin{tabular}{|c|c|}
\hline \multicolumn{2}{|c|}{ Zigbee frame structure and size } \\
\hline Frame manage & 3 bytes \\
\hline succession number & 2 bytes \\
\hline tackle information & 24 bytes \\
\hline
\end{tabular}

Table 4. Result Analysis

\begin{tabular}{|c|c|}
\hline \multicolumn{2}{|c|}{ Variable payload - if string “abnormal heart beat * is transmitted $\mathbf{- 7} * \mathbf{2 0}=\mathbf{1 4 0}$ bits= 18B } \\
\hline FCS & 2 bytes \\
\hline Total & 43 bytes \\
\hline Delay & 26.24 microseconds \\
\hline
\end{tabular}

From the calculation of delay associated, it is clear that the delay depends on packet size. The packet size depends upon the number of fields on the underlying protocol frame structure. By removing unnecessary fields on the frame the overall delay can be reduced. Modifying the existing protocol can produce considerable reduction in delay and shown in table 2.

Table 6. RIME address of the Motes

\begin{tabular}{|c|c|}
\hline RIME ADDRESS & MOTE \\
\hline 1.0 & Mote interfaced with PIR sensor. \\
\hline 95.158 & Mote interfaced with Heart beat sensor. \\
\hline 3.0 & Mote interfaced with moisture sensor. \\
\hline 2.0 & Repeater Mote. \\
\hline 38.242 & Sink Mote interfaced with PC. \\
\hline
\end{tabular}




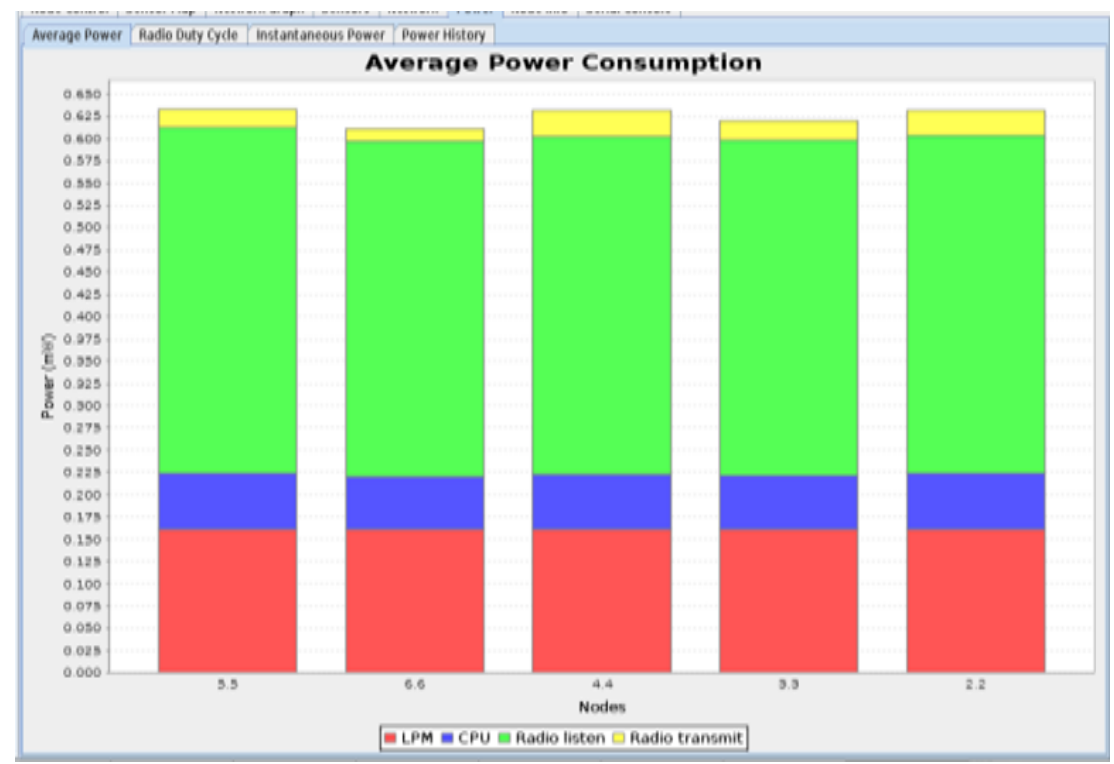

Fig 5. Simulated result Average Power Consumption Graph

\section{Conclusion}

A WBAN to endlessly watch the health conditions of patients was established using TELOSB motes. Sensors like heart-beat sensor, liquid Moisture sensor and Pyro-electric infrared (PIR) sensor were interfaced with the motes which senses data like heart-beat, moisture level, movement abnormality of the patient. The sensed data are transmitted through the established nodes which act as routers. The sink mote interfaced to the personal Work station which collects the data from the nearby repeater displays the results on its terminal. The future enhancements of this work could be developing a cross-link communication protocol interacting with network and MAC layer that improves the efficiency through optimization of power consumption, reduction of packet traversal delay, delay and maximized throughput.

\section{References}

[1] Adam Dunkels “The Contiki MAC Radio Duty Cycling Protocol” SICS Technical Report ISSN 11003154, Dec 2011

[2] Hansen, M. S. (2006). Practical evaluation of IEEE 802.15. 4/ZigBee medical sensor networks (Master's thesis, Institutt for elektronikk og telekommunikasjon).J. Zheng and Myung J. Lee, "A comprehensive performance study of IEEE 802.15.4," Sensor Network Operations Book, IEEE Press, Wiley Interscience, Chapter 4, pp. 218-237, 2006.

[3] Akyildiz, I. F., Su, W., Sankarasubramaniam, Y., \& Cayirci, E. (2002). A survey on sensor networks. IEEE Communications magazine, 40(8), 102-114.

[4] J.S. Lee , "Performance evaluation of IEEE 802.15.4 for low-rate wireless personal area networks," IEEE Transactions on Consumer Electronics, Volume 52, Pages 742- 749, August 2006.

[5] Buettner, M., Yee, G. V., Anderson, E., \& Han, R. (2006, October). X-MAC: a short preamble MAC protocol for duty-cycled wireless sensor networks. In Proceedings of the 4th international conference on Embedded networked sensor systems (pp. 307-320). 
[6] Muhammad Mahta b, olivier Berder, Daniel Menard, and olivier Sentieys "TAD-MAC : traffic-aware dynamic MAC protocol for wireless body area sensor networks" IEEE journal on emerging and selected topics in circuits and systems, vol 2, NO.1, march 2012

[7] S. Sharma, A. L. Vyas, B. Thakker, D. Mulvaney and S. Datta, "Wireless Body Area Network for health monitoring," 2011 4th International Conference on Biomedical Engineering and Informatics (BMEI), Shanghai, 2011, pp. 2183-2186.

[8] E. Dolatabadi and S. Primak, "Ubiquitous WBAN-based Electrocardiogram monitoring system," 2011 IEEE 13th International Conference on e-Health Networking, Applications and Services, Columbia, MO, 2011, pp. 110-113.

[9] Chris otto, Aleksandar milenković, Corey sanders, Emil jovanov, "System Architecture of a Wireless Body Area Sensor Network for Ubiquitous health monitoring", Journal of Mobile Multimedia, Vol. 1, No.4, 2006, PP. 307-326

[10] Mark A. Hanson, Harry C. Powell Jr., Adam T. Barth, Kyle Ringgenberg, Benton H. Calhoun, James H. Aylor, and John Lach, "Body Area Sensor Networks Challenges and Opportunities", IEEE Computer Society, Vol.42, Issue 1, January 2009, PP. 58-65

[11] Joonyoung Jung, Kiryong Ha, Jeonwoo Lee, "Wireless Body Area Network in a Ubiquitous Healthcare System for Physiological Signal Monitoring and Health Consulting", International Journal of Signal Processing, Image Processing and Pattern Recognition, volume 1, pp. 47-54 